\title{
Complex band structures and effective medium descriptions of periodic acoustic composite systems
}

\author{
Xianyu Ao and C. T. Chan \\ Department of Physics, Hong Kong University of Science and Technology, Clear Water Bay, Kowloon, Hong Kong, China
}

(Received 11 August 2009; revised manuscript received 13 October 2009; published 10 December 2009)

\begin{abstract}
We examine the complex band structures and effective medium descriptions of a periodic acoustic composite system. It is shown that if the system exhibits a negative velocity band, the assignment of the system as a phononic crystal or as a "double negative" metamaterial is unambiguous only in some cases. An example is given where the system properties can be tuned gradually and continuously from an acoustic metamaterial to a phononic crystal, and there is no sharp dividing line between these two regimes.
\end{abstract}

DOI: $10.1103 /$ PhysRevB.80.235118

PACS number(s): 78.20.Ci, 43.20.+g, 43.35.+d

In some electromagnetic (EM) metamaterials ${ }^{1}$ and photonic crystals, ${ }^{2}$ EM waves bend negatively and consequently a negative index of refraction can be assigned to such materials. The origin of negative refractive index of metamaterials is usually interpreted as the permittivity and permeability are negative simultaneously (so called "double negative") and that of photonic crystals is usually attributed to band folding. Most metamaterials are realized as periodic arrays of resonating units, and their wave properties can be described using band structures just as the case of photonic crystals. In both cases, their band structures can carry bands with negative slope. The key distinction between the two systems is that the former can be homogenized, in the sense that effective constitutive parameters can be meaningfully extracted and used, whereas the latter cannot. However, there is controversy on whether a periodic composite can be homogenized. ${ }^{3,4}$

Recent works demonstrated that a two-dimensional (2D) photonic crystal with high-permittivity dielectric rods exhibits double negative behavior. ${ }^{5,6}$ It is interesting whether a distinct boundary exists between the two regimes of photonic crystals and EM metamaterials, if one changes the permittivity gradually. The 2D wave equations for acoustic waves and EM waves are equivalent, with the mapping of variables as $\left[E_{y}, H_{x}, H_{z}, \epsilon_{y}, \mu_{x}, \mu_{z}\right] \leftrightarrow\left[p,-v_{z}, v_{x}, \kappa^{-1}, \rho_{z}, \rho_{x}\right]$ (with pressure $p$, particle velocity $v$, bulk modulus $\kappa$, and mass density $\rho)$. A high-permittivity dielectric rod in EM waves is equivalent to a low-modulus rod in acoustics and it has been demonstrated that an acoustic composite consisting of lowmodulus inclusions exhibits negative $\kappa$ and $\rho$ simultaneously. ${ }^{7}$ There are also other means to create negative $\kappa$ or $\rho$ separately. ${ }^{8}$ Phononic crystal is the counterpart of photonic crystal.

In this paper we construct an acoustic model system which is tuned gradually by changing one single parameter. As the change in parameter changes the system from a metamaterial to a phononic crystal, the description of homogenization goes from nearly perfect to marginally meaningful, but there is no sharp transition. There exists an intermediate regime where the system properties are somewhere between acoustic metamaterials and phononic crystals, and the assignment to either one category would become somewhat arbitrary. The general conclusion reported here holds true for EM waves.
We consider 2D acoustic composites consisting of a square lattice of fluid cylinders. There are frequency regions (band gaps) where the wave is evanescent so the wave number is complex. Basically there are two types of band gaps, one is from Bragg-like multiple scattering, and the other is from the coupling of resonators. We shall examine their complex band structures and see if there are obvious differences that can distinguish the two origins of gaps. The complex band structure is a relation between the complex wave number (wave vector) and the frequency. The complex band structure can be calculated by the layer-multiple scattering method (L-MST) ${ }^{9,10}$ We will also show results calculated by the multiple scattering method (MST) (Ref. 11) to check the consistency for pass bands. In the L-MST method, we show the smallest nonzero value of the imaginary values of the $k$ vector inside the gaps.

The band structures are typically displayed using the reduced zone scheme. If we want to assign a negative refractive index to a system that exhibits a negative group velocity band, it is meaningful only if the wave function has strong amplitude in the first Brillouin zone (BZ). It may not be the case if the negative band is a simple geometric artifact of zone folding. This can of course be achieved by examining the Bloch wave functions. Alternatively, we can use effective medium theory (EMT) as a guide. If band dispersions coming from effective medium parameters agree quantitatively with the exact band structures near the zone center, then the assignment of a negative index is by definition meaningful. In particular, any material that can be claimed to be a metamaterial should imply that the effective medium parameters provide a quantitative description near the zone center. We thus put emphasis on comparing the exact band structures with EMT results.

Following the EMT in Ref. 12 (assuming that the scattering of the cylinder is dominated by its monopolar and dipolar channels), we obtain the expressions which relate the frequency-dependent effective bulk modulus $\kappa_{e}(\omega)$, effective mass density $\rho_{e}(\omega)$, and effective wave number $K_{e}(\omega)$ as

$$
\frac{\rho_{e}}{\rho_{0}} \frac{k_{0}}{K_{e}} \frac{J_{m}\left(K_{e} r_{0}\right)}{J_{m}^{\prime}\left(K_{e} r_{0}\right)}=\frac{J_{m}\left(k_{0} r_{0}\right)+D_{m} H_{m}^{(1)}\left(k_{0} r_{0}\right)}{J_{m}^{\prime}\left(k_{0} r_{0}\right)+D_{m} H_{m}^{(1)^{\prime}}\left(k_{0} r_{0}\right)}, \quad m=0,1,
$$




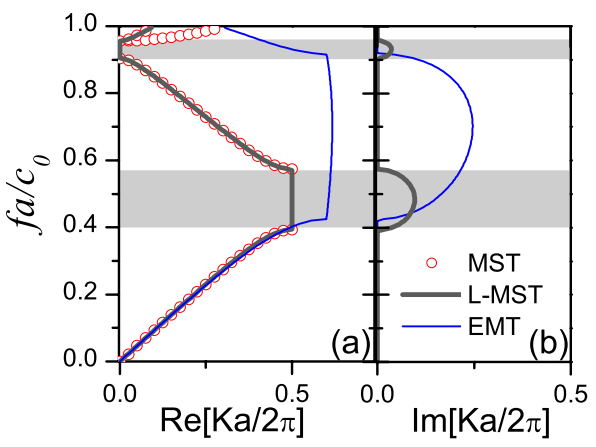

FIG. 1. (Color online) Complex band structure along $\Gamma$-X direction for a square lattice of cylinders (with radius $r_{s}=0.25 a$, mass density $\rho_{s}=20000 \mathrm{~kg} / \mathrm{m}^{3}$, and longitudinal velocity $c_{s}=5000 \mathrm{~m} / \mathrm{s}$ ) in water, (a) the real part, and (b) the imaginary part. Only the nondeaf modes (those excitable by normal incidence due to symmetry) are shown for L-MST. The frequency gaps are shaded in light gray.

$$
K_{e}=\omega \sqrt{\rho_{e} / \kappa_{e}}
$$

where $\pi r_{0}^{2}=a^{2}$ ( $a$ is lattice constant) and $D_{m}$ is the Mie scattering coefficients for angular momentum channel $m$. The wave number in the host is $k_{0}=\omega \sqrt{\rho_{0} / \kappa_{0}}$, where $\rho_{0}$ and $\kappa_{0}$ are the mass density and bulk modulus of the fluid host. $J_{m}(x)$ and $H_{m}^{(1)}(x)$ are the Bessel function and Hankel function of the first kind, respectively. For a cylinder with radius $r_{s}$, mass density $\rho_{s}$ and bulk modulus $\kappa_{s}=\rho_{s} c_{s}^{2}\left(c_{s}\right.$ denotes the longitudinal velocity), the Mie coefficients can be found in Ref. 13. When $K_{e} r_{0} \ll 1$ with the small argument approximation ${ }^{12}$ for $J_{m}\left(K_{e} r_{0}\right)$ and $J_{m}^{\prime}\left(K_{e} r_{0}\right)$ in Eq. (1), we can further see that $\kappa_{e}(\omega)$ and $\rho_{e}(\omega)$ are directly related to monopolar $(m=0)$ and dipolar $(m=1)$ channels, respectively. Instead of the small argument approximation, we solve Eq. (1) directly by searching complex roots to obtain $\rho_{e}(\omega), \kappa_{e}(\omega)$, and $K_{e}(\omega)$.

In the following, we will first discuss two model systems that have spectral gaps. In the first case (Fig. 1), the gaps are due to Bragg scattering, and in the second case (Fig. 2), the gaps are due to resonances. We will show that the two types of gaps can be distinguished by examining the trajectory of

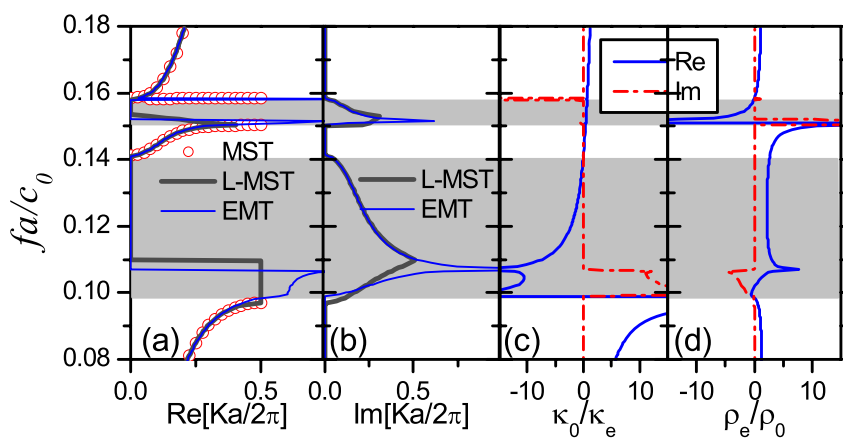

FIG. 2. (Color online) Complex band structure along $\Gamma$-X direction for a square lattice of cylinders (the same cylinder as in Fig. 1 but with $c_{s}=100 \mathrm{~m} / \mathrm{s}$ ) in water, (a) the real part, and (b) the imaginary part. (c) Effective bulk modulus $\kappa_{0} / \kappa_{e}$. (d) Effective mass density $\rho_{e} / \rho_{0}$. The frequency gaps are shaded in light gray. the real and imaginary part of the wave vector inside the gaps.

Figure 1 shows the complex band structure for a square lattice of fluid cylinders (with $r_{s}=0.25 a, \rho_{s}=20000 \mathrm{~kg} / \mathrm{m}^{3}$, and $c_{s}=5000 \mathrm{~m} / \mathrm{s}$ ) in water (with $\rho_{0}=1000 \mathrm{~kg} / \mathrm{m}^{3}$, and $c_{0}$ $=1490 \mathrm{~m} / \mathrm{s}$ ). In high- $c_{s}$ systems, the lowest frequency gaps are typically the consequence of Bragg scattering. Inside the gap the $k$ vector is a complex number $K a=\pi+i K_{i}$ characterized by a real part pinned at the zone boundary and an imaginary part $K_{i}$ that is continuous and smooth across the whole gap, with a maximum value near the mid gap. Such characteristics are indeed verified by the complex band structure calculated by L-MST (thick lines). The regions of the Bragg gaps are shaded by light gray color. For comparison, we extracted the dispersion curves $K_{e}(\omega)$ from EMT according to Eq. (1) (shown as thin lines). The EMT results can reproduce very well the dispersion of the first band $(\operatorname{Re}[K a / 2 \pi]$ $<0.5)$, and this agreement is consistent with the general argument that the first band is in the first BZ in the extended zone scheme. EMT breaks down completely beyond the first band, as expected. We thus note that a typical Bragg gap is characterized by the pinning of the real part of $\mathrm{Ka}$ at the zone boundary throughout the gap, a continuous and smooth variation of the imaginary part of $K a$, and the failure of EMT to describe quantitatively the dispersion near the gap.

Now turn to typical resonance gaps, which occur as a result of the hybridization between the flat bands (originating from coupling of resonant modes of individual cylinders) and the continuum bands (corresponding to longitudinal propagation in an effective homogeneous medium). ${ }^{14}$ The model system is the same as that shown in Fig. 1, but with $c_{s}=100 \mathrm{~m} / \mathrm{s}$. The slower wave speed means shorter wavelength inside the cylinder which induces Mie resonances at lower frequencies. The complex band structure calculated by L-MST is plotted as thick lines in Fig. 2(a). The two resonance gaps are shaded in light gray. The first gap can be traced to a monopolar Mie resonance of the single cylinder and the second gap corresponds to a dipolar Mie resonance. The real part of the complex wave number is $\operatorname{Re}[K a]=\pi$ for the lower frequency part of both gaps, but jumps to $\operatorname{Re}[K a]=0$ at a certain frequency inside the gap. Similar features were reported in Ref. 15. The real part of $K a$ has a sudden jump inside both resonance gaps, in contrast to the pinning of $K a$ at the zone boundary for Bragg gaps. In Fig. 2(b) the imaginary part of the complex band structure is shown as thick lines. While the curve is continuous, the trajectory has a cusp at which the slope changes abruptly at the frequency where the real part jumps from the zone boundary to the zone center. This is in contrast to the smooth variation in $\operatorname{Im}[K a]$ for Bragg gaps. The dispersion curves $K_{e}(\omega)$ from EMT [Eq. (1)] are plotted as thin lines for comparison with the exact band structures in Figs. 2(a) and 2(b) for the real part and the imaginary part respectively. In contrast to the failure of EMT after the first band in the case of Bragg gaps (Fig. 1), EMT now gives the correct salient features. In particular, EMT results exhibit the abrupt change in $\operatorname{Re}[\mathrm{Ka}]$ and the discontinuity of the slope of $\operatorname{Im}[K a]$, although the frequency at which this happens is slightly off in the first gap. This is not surprising since EMT is not expected to work quantitatively very close to a resonance and far away from 


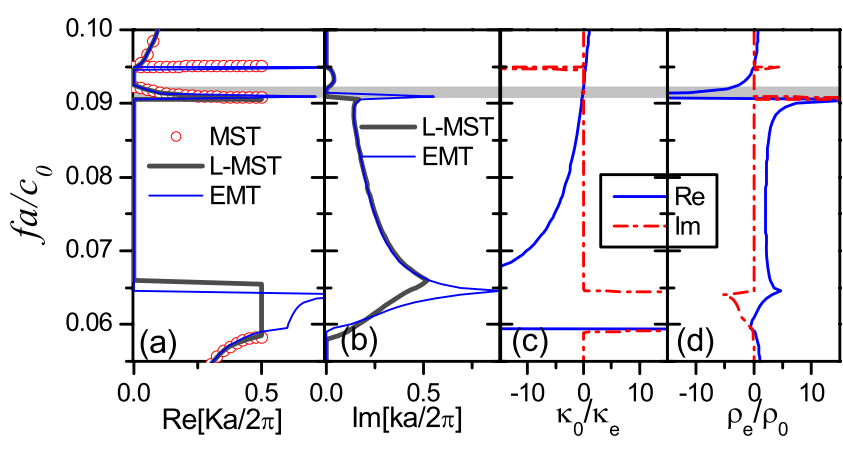

FIG. 3. (Color online) The same as Fig. 2 but with $c_{s}$ $=60 \mathrm{~m} / \mathrm{s}$. The frequency region with double negativity is shaded in light gray.

zone center. In particular, the EMT results can reproduce quantitatively the dispersion of all the pass bands $(\operatorname{Re}[K a / 2 \pi]<0.4)$. The fact that EMT gives a quantitative description for the pass bands indicates that for these pass bands wave functions have strong amplitudes inside the first $\mathrm{BZ}$, and thus the assignment of effective parameters are meaningful and the wave properties can be meaningfully called "metamaterial" properties. The flat band near $f a / c_{0}$ $=0.16$ is for transverse modes which appear when $\rho_{e}=0$ (deaf band).

The effective bulk modulus $\kappa_{0} / \kappa_{e}$ and effective mass density $\rho_{e} / \rho_{0}$ extracted using EMT [Eq. (1)] are shown in Figs. 2(c) and 2(d). Inside the lower frequency gap, the real part of $\kappa_{e}$ is negative, while the real part of $\rho_{e}$ is positive. This frequency region corresponds to a negative modulus system giving rise to a forbidden gap (consistent with the monopolar resonance). In the higher frequency gap, the real part of $\kappa_{e}$ is positive, while the real part of $\rho_{e}$ is negative. This frequency region corresponds to a negative density system giving rise to a forbidden gap, consistent with the dominance of the dipolar response in this regime. We note that the effective parameters from Eq. (1) are real when the system is lossless. The imaginary part of $\kappa_{0} / \kappa_{e}$ and $\rho_{e} / \rho_{0}$ (of opposite signs) inside the frequency regions with $\operatorname{Re}[K a]=\pi$ should not be interpreted as loss or gain, and this corresponds to the socalled resonance-antiresonance coupling in Ref. 16. This actually manifests the necessity for EMT to break down in the frequency region near the zone boundary, where the waves see periodic structures, and very close to the resonance, where the effective wavelength is small. The effective parameters from Eq. (1) quantitatively agree with those obtained by a retrieval procedure. ${ }^{16}$

The negative modulus (monopolar) and negative density (dipolar) resonances gaps are separated in frequency in the system shown in Fig. 2. We now reduce the speed inside the cylinder further to $c_{s}=60 \mathrm{~m} / \mathrm{s}$ (see Fig. 3). This will bring down the frequency of both resonances, but now the two resonances overlap over a certain range of frequencies. Compared with Fig. 2(a) we see that the new feature in Fig. 3(a) is a band with negative slope (the light gray shaded area). This manifests that double negativity gives rise to negative bands in acoustic metamaterials. ${ }^{7}$ The coincidence of monopolar and dipolar resonances should be the key reason for the "double negativity." We also see that EMT can reproduce

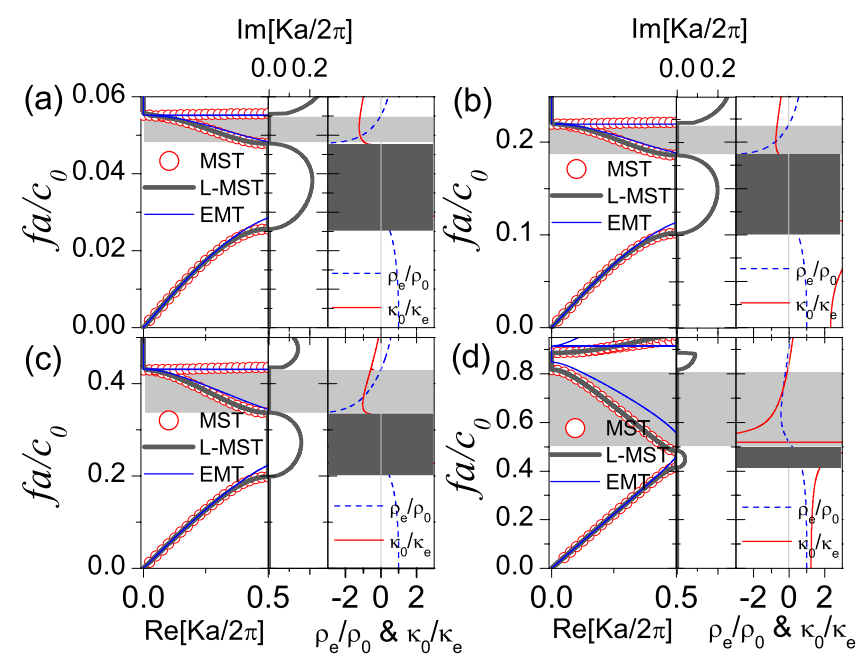

FIG. 4. (Color online) A square lattice of cylinders in water, with longitudinal velocity of the cylinders as (a) $c_{s}=50 \mathrm{~m} / \mathrm{s}$, (b) $c_{s}=200 \mathrm{~m} / \mathrm{s}$, (c) $c_{s}=400 \mathrm{~m} / \mathrm{s}$, (d) $c_{s}=1000 \mathrm{~m} / \mathrm{s}$. The radius of cylinders is $r_{s}=0.25 a$. Left panel is the real part of the complex band structure. Middle panel is imaginary part of the complex band structure from L-MST. Right panel is $\kappa_{0} / \kappa_{e}$ and $\rho_{e} / \rho_{0}$ from EMT. The values of $\kappa_{0} / \kappa_{e}$ in (a) and (b) are divided by 50 and 5 , respectively.

well this negative band, and thus this band is in the first BZ in the extended zone scheme. That is to say, this negative band appears as a result of both effective bulk modulus and effective mass density are negative [see Figs. 3(c) and 3(d)]. The flat band above the negative band again corresponds to $\rho_{e}=0$.

In photonic/phononic crystals, the effect of periodicity is emphasized and the existence of negative slope bands is attributed to Bragg scattering, while in metamaterials, the effect of resonance is emphasized. Below we construct an acoustic model whose properties can be tuned continuously from "double negative" acoustic metamaterials to phononic crystals. The system is again a simple square array of cylinders with $r_{s}=0.25 a$. The density of the cylinder is assumed to be the same as that of water for simplicity. The longitudinal wave velocity of the cylinder, $c_{s}$, is the only parameter that is changed. It is increased gradually to monitor the change in their band structures, and the agreement with EMT results.

The results are summarized in Fig. 4. The left and middle panels show the complex band structures, with the open circles calculated by MST, thick lines by L-MST, and thin lines are for dispersion curves from EMT [Eq. (1)]. The right panels show $\kappa_{0} / \kappa_{e}$ and $\rho_{e} / \rho_{0}$ extracted from EMT (real part only). Since EMT has limited validity in the frequency region with $\operatorname{Re}[K a]=\pi$ as discussed above, we do not show the effective parameter results given by EMT in this frequency region (shaded by dark gray). The value of $c_{s}$ varies from a low value of $c_{s}=50 \mathrm{~m} / \mathrm{s}$ in Fig. 4(a) to a moderate value of $c_{s}=1000 \mathrm{~m} / \mathrm{s}$ in Fig. 4(d). Regardless of the scales of the vertical axes in Figs. 4(a)-4(d), the first two bands have similar features, with the first band having a positive slope and the second band having a negative slope, separated by a gap. Inside this gap, the imaginary part of the complex wave number is always smooth and continuous, similar to 
the one shown in Fig. 1(b), and the real part is $\operatorname{Re}[K a]=\pi$. This is true for all four cases, even for cases like Fig. 4(a) where resonances dominate the physics.

We would like to compare Fig. 4(a) with Fig. 3(a). The coincidence of resonances leads to a negative band in both systems. However, in Fig. 3(a), the gap below the negative band has the characteristic of a resonance gap, but in Fig. 4(a), the gap below the negative band has the characteristic of a Bragg gap. We thus cannot use the gap characteristic to judge unambiguously whether we have a "double negative" medium.

In Figs. 4(a)-4(c), the effective dispersion curves from EMT can reproduce quantitatively all the bands with $\operatorname{Re}[K a / 2 \pi]<0.3$. In addition, $\kappa_{e}(\omega)$ and $\rho_{e}(\omega)$ shown in the right panels exhibit simultaneous negative values in the frequency range where the negative band exists (the light gray shaded areas). In these situations, the EMT gives quantitative description, meaning that the effective parameters are physically meaningful, and in addition, the negative band is the consequence of "double negativity." We note that the third band, which is just above the negative band, is nearly nondispersive for Fig. 4(a), but there is a noticeable dispersion of this band in Fig. 4(d). Examining the effective parameters, shown in the right panels of Figs. 4(a) and 4(d), we found that the existence of the flat band coincides with $\rho_{e}=0$, which allows for a transverse mode solution. In Fig. 4(d) the effective dispersion curve can reproduce quantitatively only the first band with $\operatorname{Re}[\mathrm{Ka} / 2 \pi]<0.4$. Although there is a frequency region in which $\kappa_{0} / \kappa_{e}$ and $\rho_{e} / \rho_{0}$ given by EMT are both negative, the corresponding effective dispersion curve does not agree with the band structure. The absence of the flat band due to the zero effective parameter is another feature that distinguishes Fig. 4(a) from the situation in Fig. 1(a).

We can of course go to even higher values of $c_{s}$, and the deviation of the effective parameters description will be even more conspicuous. However, the point we want to emphasize here is that the transition from $c_{s}=50 \mathrm{~m} / \mathrm{s}$ to $c_{s}$ $=1000 \mathrm{~m} / \mathrm{s}$ is in fact a rather smooth transition. There is a lack of a really sharp dividing line. One useful yardstick would be the use of EMT. If effective parameters, as extracted from Eq. (1), can reproduce quantitatively the negative bands near the zone center, then the system can be safely referred to as a "double negative metamaterial." The existence of flat bands that can be traced to zero values of effective mass density is another characteristic for acoustic waves (but not necessarily for elastic waves). We may say that the distinction between acoustic metamaterials and phononic crystals are unambiguous only in certain cases such as those in Figs. 4(a) and 4(d), at least in this model system we choose to illustrate the physics. There is always an intermediate regime in which the distinction is blurred. Even for the situation in Fig. 4(d), which corresponds to a typical phononic band gap system, the EMT results are still qualitatively correct to some extent. In this particular geometry, the lowest gap originates from the $m=0$ scattering, and the Bragg gap does not deviate too much from the $m=0$ Mie resonance of a single cylinder. But as the effective wavelength is no longer larger than the size of the inclusion, the dispersion of the negative band cannot be given correctly by EMT, as shown in Fig. 4(d). The effective medium only knows about the scattering of a single scatterer in an averaged background and cannot predict collective (Bragg) scattering effect, and as such, the gap size is wrong.

The physics is also tied to the length scale. When the background wavelength is much larger than the lattice constant, homogenization of the composite to extract effective parameters is physically meaningful. The negative-slope band in Fig. 4(a) corresponds to $a / \lambda \approx 0.05$, we can well assign this case to a regime of double negative metamaterial. In the opposite regime of phononic crystals, as in the cases of Fig. 4(d), $a$ and $\lambda$ are of the same order, and the negative effective parameters extracted by EMT start to lose those useful physical meaning. In Figs. 4(b) and 4(c), EMT can predict reasonable results. When $c_{s}$ of the cylinders is increased gradually as in Figs. 4(a)-4(d), the negative-slope band moves to a higher-frequency range and the composite changes from a double negative metamaterial to a phononic crystal continuously.

In conclusion, we examined the characteristics of band structures for phononic crystals and acoustic metamaterials based on a model with 2D cylinders in water and compared results with parameters extracted using the effective medium theory. Resonance gaps and Bragg gaps possess distinguishable complex band structures. However, when two types of Mie resonances are combined to form double negative acoustic metamaterials, no universal feature can be found for gaps near the resulting double negative band. We further considered a model where the system can be tuned gradually by changing just one parameter, and the system transforming gradually from an acoustic metamaterial to a phononic crystal. There is no distinct boundary between these two regimes.

This work is supported by Hong Kong RGC (Grant No. 600305).
${ }^{1}$ D. R. Smith, W. J. Padilla, D. C. Vier, S. C. Nemat-Nasser, and S. Schultz, Phys. Rev. Lett. 84, 4184 (2000).

${ }^{2}$ M. Notomi, Phys. Rev. B 62, 10696 (2000).

${ }^{3}$ T. Koschny, P. Markos, D. R. Smith, and C. M. Soukoulis, Phys. Rev. E 68, 065602(R) (2003).

${ }^{4}$ A. L. Efros, Phys. Rev. E 70, 048602 (2004).

${ }^{5}$ L. Peng, L. Ran, H. Chen, H. Zhang, J. A. Kong, and T. M.
Grzegorczyk, Phys. Rev. Lett. 98, 157403 (2007).

${ }^{6}$ K. Vynck, D. Felbacq, E. Centeno, A. I. Cabuz, D. Cassagne, and B. Guizal, Phys. Rev. Lett. 102, 133901 (2009).

${ }^{7}$ J. Li and C. T. Chan, Phys. Rev. E 70, 055602(R) (2004).

${ }^{8}$ Z. Liu, X. Zhang, Y. Mao, Y. Y. Zhu, Z. Yang, C. T. Chan, and P. Sheng, Science 289, 1734 (2000); N. Fang, D. Xi, J. Xu, M. Ambati, W. Srituravanich, C. Sun, and X. Zhang, Nat. Mater. 5, 
452 (2006); Y. Ding, Z. Liu, C. Qiu, and J. Shi, Phys. Rev. Lett. 99, 093904 (2007); X. Y. Ao and C. T. Chan, Phys. Rev. E 77, 025601(R) (2008).

${ }^{9}$ N. Stefanou, V. Yannopapas, and A. Modinos, Comput. Phys. Commun. 113, 49 (1998).

${ }^{10}$ L. C. Botten, N. A. Nicorovici, R. C. McPhedran, C. Martijn de Sterke, and A. A. Asatryan, Phys. Rev. E 64, 046603 (2001).

${ }^{11}$ N. A. Nicorovici, R. C. McPhedran, and L. C. Botten, Phys. Rev. E 52, 1135 (1995).
${ }^{12}$ Y. Wu, J. Li, Z.-Q. Zhang, and C. T. Chan, Phys. Rev. B 74, 085111 (2006).

${ }^{13}$ Y.-Y. Chen and Z. Ye, Phys. Rev. E 64, 036616 (2001).

${ }^{14}$ R. Sainidou, N. Stefanou, and A. Modinos, Phys. Rev. B 66, 212301 (2002).

${ }^{15}$ C. Tserkezis, N. Papanikolaou, G. Gantzounis, and N. Stefanou, Phys. Rev. B 78, 165114 (2008).

${ }^{16}$ T. Koschny, P. Markos, E. N. Economou, D. R. Smith, D. C. Vier, and C. M. Soukoulis, Phys. Rev. B 71, 245105 (2005). 